\title{
Amifostine enhancement of the anti-cancer effects of paclitaxel in endometrial cancer is TP53-dependent
}

\author{
WENTAO LUO ${ }^{1}$, FAN WU $^{2}$, RUBA ELMAOUED ${ }^{3}$, BRANDON B. BECK ${ }^{4}$, EDGAR FISCHER $^{5}$, \\ XIANGBING MENG ${ }^{4}$, KIMBERLY K. LESLIE ${ }^{4}$ and DONGHAI DAI ${ }^{4}$
}

\begin{abstract}
${ }^{1}$ Department of Internal Medicine, University of New Mexico, Albuquerque, NM 87131; ${ }^{2}$ Research Center, University of Virginia, Charlottesville, VA 22908; ${ }^{3}$ Department of Anesthesiology, Upstate Medical University, Syracuse, NY 13210; ${ }^{4}$ Department of Obstetrics and Gynecology, University of Iowa Health Care, Iowa City,

IA 52242; ${ }^{5}$ Department of Pathology, University of New Mexico, Albuquerque, NM 87131, USA
\end{abstract}

Received June 24, 2010; Accepted July 30, 2010

DOI: 10.3892/ijo_00000770

\begin{abstract}
Endometrial cancer (ECa) is the fourth most common malignancy in women. Currently, there is no effective therapy for advanced and recurrent cancer. Among the pooroutcome endometrial cancers, there is a high frequency of TP53 mutations. We have previously reported that amifostine has a direct anti-cancer effect and has a significant synergistic effect with paclitaxel when used in endometrial cancer cell and xenograft models. In this report, using a cell line with knock-down p53 expression through siRNA, we found that amifostine enhancement of paclitaxel's anticancer effect is p53 status-dependent. Amifostine promotes entry into the G2-M phase through regulation of cyclin-dependent kinase-1 activity in cells with dysfunctional p53, thereby enhancing cancer cell sensitivity to paclitaxel. The synergistic effect between amifostine and paclitaxel was further confirmed in vivo using xenografts created with primary patient tumor tissue. Sensitivity to the therapeutic effect of paclitaxel in combination with amifostine was dependent upon the status of p53. A tumor with a nonsense TP53 mutation showed increased therapeutic response to paclitaxel and amifostine as measured by tumor weight compared to a tumor with wildtype TP53. Our study provides a rationale for a clinical trial of combined paclitaxel and amifostine in endometrial cancer patients whose tumors harbor TP53 mutations.
\end{abstract}

Correspondence to: Dr Donghai Dai, Department of Obstetrics and Gynecology, University of Iowa Health Care, 500 Newton Road, Iowa City, IA 52242, USA

E-mail: donghai-dai@uiowa.edu

Abbreviations: ECa, endometrial cancer; UEC, uterine endometrioid endometrial cancer; UPSC, uterine papillary serous carcinoma; PTX, paclitaxel; AMF, amifostine

Key words: amifostine, paclitaxel, endometrial cancer, TP53, primary tumor xenograft

\section{Introduction}

Endometrial cancer (ECa) is the fourth most common malignancy in women with 42,160 new cases and 7,780 deaths expected in 2009 (1). Estrogen overexposure has been thought to be an important etiological factor for the development of ECa (2). ECa related to estrogen overexposure typically develops via a pathway with a sequence of hyperplastic changes of the endometrium with increasing premalignant potential (3). These tumors typically are well-to-intermediately differentiated in morphology and are often diagnosed at an early stage, and thus they have an excellent prognosis with a 5 -year survival rate of $85.6 \%$ (3). This group of tumors is described as type I ECa. The majority of type I ECa is endometrioid endometrial carcinoma (UEC), the most common histological type with an incidence of approximately $70-80 \%$ of total ECa (4). The second type of ECa develops from an atrophic or rarely cycling endometrium, which is not associated with the classical risk factors for ECa (3). These type II ECa are mostly of a high grade or have high-risk histology such as uterine papillary serous carcinoma (UPSC). UPSC is a rare aggressive endometrial tumor characterized by late stage at presentation, intra-peritoneal expansion and poor prognosis (3). UPSC is similar to serous papillary ovarian cancer in presentation and outcome. UPSC accounts for 5-10\% of endometrial cancer cases but they are responsible for $50 \%$ of all treatment failures $(3,5,6)$. Regardless of the type of treatment, more than $50 \%$ of cases recur shortly (7). The prognosis for UPSC is very poor with a 5-year survival rate of $45-62 \%$ for stage I-II and $11 \%$ for stage III-IV $(6,8)$.

Overall, $20 \%$ of endometrial cancers have a TP53 mutation (9) but the mutation rate could be as high as $90 \%$ in UPSC $(10,11)$. Mutation of TP53 was detected in $78 \%$ of endometrial intraepithelial carcinomas, presumably a precursor of UPSC, suggesting that TP53 gene mutation is an early event in UPSC carcinogenesis (10). It was believed that UPSC is a TP53 mutation-driven neoplasm and this group of tumors bypasses the slow hormone-dependent pathway of tumorigenesis but instead undergoes early TP53 alterations that lead to rapid tumor development $(10,11)$. We reported previously that amifostine enhances paclitaxel's therapeutic effect in endometrial 
cancer in addition to its protective effect in peripheral blood cells (12). In this study, we investigated the p53-dependent mechanism of synergistic effects between amifostine and paclitaxel in two cell lines and two patient tumors representative of the two types of endometrial cancer.

\section{Materials and methods}

Cells and reagents. Ishikawa-H cell $(\mathrm{H})$ has been used in previous studies (13-15). They are maintained in DMEM with $10 \% \mathrm{FBS}$ in $95 \% \mathrm{O}_{2}$ and $5 \% \mathrm{CO}_{2}$ at $37^{\circ} \mathrm{C}$. Amifostine (Ethyol, MedImmune Oncology, Inc., Gaithersburg, MD) was purchased from the University of New Mexico Hospital pharmacy. Paclitaxel for the in vitro studies was purchased from Sigma (St. Louis, MO) and reconstituted in DMSO. Paclitaxel (Taxol, Mead Johnson, Princeton, NJ) for the in vivo study was purchased from the University of New Mexico Hospital pharmacy and further diluted in saline before i.v. administration.

Creation of the H-p53 ${ }^{K D}$ cell line. DH5 $\alpha$ E. coli bacteria cells of Subcloning Efficiency (Invitrogen Corporation, Carlsbad, CA) were transformed with $1 \mu 1$ of plasmid carrying p53 short hairpin RNA (shRNA). The pHYGU6 vector was purchased from Ambion ${ }^{\circledR}$ (Austin, TX). The p53 shRNA targets TP53 coding sequences 775 to 794 . This sequence was cloned into the pHygromycin/U6 plasmid using HindIII and BamHI restriction enzymes. This 4885 base pair (bp) plasmid carries a hygromycin marker. Ishikawa $\mathrm{H}$ cells were transfected using the GenePorter system per the instructions provided. Confirmation of p53 silencing was assessed by Western blot analysis (data not shown).

Calculation of the $I C_{50}$ of paclitaxel and amifostine. Cells were exposed to varying concentrations of paclitaxel (2$1,000 \mathrm{nmol} / \mathrm{l})$, amifostine $(28 \mu \mathrm{mol} / \mathrm{l}-3 \mathrm{mmol} / \mathrm{l})$ for different time periods and the $\mathrm{IC}_{50}$ values were calculated according to methods previously reported (12). Means and SEs were calculated from at least three experiments.

Flow cytometry. Cells were analyzed by a FacScan machine (Becton-Dickinson, Franklin Lakes, NJ) with the CellQuest Program version 3.3 (Becton-Dickinson) as reported previously (12).

Apoptosis study. Apoptotic cells were identified by their fragmented nuclear morphology according to the method previous reported (12). The ratio was obtained as a percentage of apoptotic cells over total cell number in one high magnification field. Means and SEs were calculated from at least three experiments.

Western blot analysis. Primary antibodies for p53, p21, Cdk1, Cdk1-T15, and B-actin were purchased from Santa Cruz Biotechnology (Santa Cruz, CA). Extracts containing $100 \mu \mathrm{g}$ of protein/lane were resolved on $12.5 \%$ SDS-PAGE gels, transferred to nitrocellulose membranes, probed with primary antibodies, and visualized using enhanced chemiluminescence as described previously (12).
Xenograft study. Protocol for collection of human endometrial tumors was approved by HRRC at the University of New Mexico. Fresh patient tumors were obtained at the Department of Pathology at the University of New Mexico immediately after their arrival at the gross room. Fat and necrotic tissues were trimmed and tumor tissues were rinsed with cell culture medium. They were minced into fine homogenate and mixed with the medium. Typically, $10 \mathrm{mg}$ of tumor tissues were mixed with 100-200 $\mu 1$ medium for injection into one mouse with three mice for each patient tumor to create the first generation xenografts. Six- to eight-week-old athymic, Crl:NU/NU-nuBR female mice were purchased from NCI Frederic, MD. The experimental protocols were approved by the University of New Mexico Health Sciences Center Institutional Animal Care and Use Committee. The first generation xenografts were used for histology analysis, TP53 DNA sequencing and further propagation into athymic mice for therapeutic studies. One to two weeks after subcutaneous injection, mice with visible tumors were selected and randomly divided into three groups to investigate the effects of paclitaxel and amifostine on tumor growth. Amifostine $(120 \mathrm{mg} / \mathrm{kg})$ was given through i.p. injection every Monday, Tuesday, and Wednesday. Paclitaxel (12 mg/kg) was given through tailvein injection $30 \mathrm{~min}$ after amifostine every Wednesday. The control received saline only. Treatment lasted for 3-4 weeks and tumors were isolated 1 week after the last dose of paclitaxel. Tumor wet weights were analyzed by one-way ANOVA followed by Holm-Sidak method for pairwise comparison.

P53 sequencing. mRNA was extracted using RNeasy Mini kit from Qiagen, Valencia, CA. cDNA was created using Advantage RT-for-PCR kit, Clontech, Palo Alto, CA. The following primers were used for PCR and Sanger sequencing. Primer pair 1: F:agtctagagccaccgtcca, R:aggtctgaaaatgtttcctg actca; pair 2, F:cagtcagatcctagcgtcgag, R:tgcaagtcacagacttgg ctgt, Pair 3, F:attctgggacagccaagtctgtga, R: aaacacgcacctcaaa gctgttcc. Pair 4, F:acagctttgaggtgcgtgtttgtg, R:gcaagcaagggtt caaagacccaa.

\section{Results}

An endometrial cancer cell line, Hec50co (CO) cell, was reported to have a papillary serous morphology in vivo and with a p53 null mutation $(15,16)$. We have shown that AMF and PTX have a synergistic effect on CO cell growth in in vitro and in vivo (12). To investigate whether AMF's modulation of PTX's activity is dependent upon p53 status, we used another endometrial cancer cell line, Ishikawa-H cells $(\mathrm{H})$ and a Ishikawa-H cell-derived stable cell line $(\mathrm{H}-$ p53 ${ }^{\text {silenced}}$ ) with p53 knocked-down created through introduction of a plasmid carrying a short-hairpin sequence targeting p53 mRNA $(17,18)$. H cells were originally derived from a 39-year-old woman with endometrial adenocarcinoma (19). The primary tumor and xenografts in mice showed a welldifferentiated feature (19). The p53 protein level in the $\mathrm{H}$ p53 $3^{\text {silenced }}$ stable cells was confirmed by Western blot to be knocked down by more than $90 \%$ (data not shown). The control $\mathrm{H}$ cell transfected with shRNA targeting GFP (H-GFP) have a similar level of p53 as the parental $\mathrm{H}$ cells (data not shown). 
Table 1. $\mathrm{IC}_{50}$ values of amifostine (AMF) and paclitaxel (PTX).

\begin{tabular}{lcc}
\hline Drug/Cell & H cells & H-p53 silenced cells \\
\hline AMF & $2.3 \pm 0.3 \mathrm{mM}$ & $1.2 \pm 0.04 \mathrm{mM}$ \\
PTX & $70.9 \pm 12 \mathrm{nM}$ & $8.8 \pm 1.65 \mathrm{nM}$ \\
PTX IC $_{50}$ if & $150.14 \mathrm{nM}$ & $2.4 \mathrm{nM}$ \\
pretreated with AMF & & \\
\hline
\end{tabular}

$\mathrm{IC}_{50}$ values of paclitaxel and amifostine alone and in combination. $\mathrm{H}$, Ishikawa $\mathrm{H}$ cells; $\mathrm{H}-\mathrm{p} 53^{\text {silenced }}$, p53 silenced Ishikawa $\mathrm{H}$ cells. Values indicated in the Table are the concentrations of the drugs that result in a $50 \%$ reduction in cell number at $24 \mathrm{~h}$.

AMF modulation of PTX $I C_{50}$ is p53 status-dependent. PTX was less effective in $\mathrm{H}$ cells than in $\mathrm{CO}$ cells with an $\mathrm{IC}_{50}$ dose 4 times greater than that in $\mathrm{CO}$ cells (Table I) (12). By pretreating with $\mathrm{AMF}, \mathrm{PTX}$ 's $\mathrm{IC}_{50}$ dose was increased by 2-fold, suggesting that $\mathrm{H}$ cells became more resistant to PTX in the presence of AMF. However, when p53 was knocked down, $\mathrm{H}$ cells became sensitive to PTX again $\left(\mathrm{IC}_{50}=8.8 \mathrm{nM}\right)$, which was further enhanced by pre-treatment of $\mathrm{AMF}\left(\mathrm{IC}_{50}=2.4 \mathrm{nM}\right)$. This suggests that AMF can effectively enhance PTX's cytotoxicity when p53-dependent pathways are disrupted.

The AMF modulation of PTX's effect on cell cycle is p53dependent. PTX induced an increase of cells in $\mathrm{G} 2 / \mathrm{M}$ phase in both $\mathrm{H}$ and $\mathrm{H}-\mathrm{p} 53^{\text {silenced }}$ cells (Fig. 1A and B), consistent with PTX-induced mitotic arrest due to its suppression of spindle dynamics (20). AMF did not have an effect on the $\mathrm{G} 2 / \mathrm{M}$ phase in $\mathrm{H}$ cells but registered a slight but significant effect in $\mathrm{H}-\mathrm{p} 53^{\text {silenced }}$ cells $(25.9 \pm 1.4 \%$ in Ctrl vs. $21.8 \pm 1.2 \%$ in $\mathrm{AMF}, \mathrm{p}<0.05$, Fig. $1 \mathrm{~A}$ and $\mathrm{B}$ ), indicating that AMF's effect is mainly in G1 when p53 was functional but it becomes significant in G2/M when p53 was disrupted. Furthermore, interactions between AMF and PTX and the cell cycle presented two different phenomena depending upon p53 status. Addition of AMF to paclitaxel in $\mathrm{H}$ cells has effectively removed PTX-induced cell accumulation in G2/M (Fig. 1A). Interestingly, when p53 was knocked down in H-p53 $3^{\text {silenced }}$ cells, PTX-induced G2/M arrest was again remarkably enhanced by the addition of AMF (Fig. 1B). To investigate the interaction of these two drugs for mitotic arrest, we measured the percentage of cells in mitosis (mitotic index) through chromatin staining by Hoechst dye and identification of typical morphology consistent with mitosis. There was no difference in mitotic index between the control and AMFtreated cells either in $\mathrm{H}$ or $\mathrm{H}-\mathrm{p} 53^{\text {silenced }}$ cells, and PTX induced a similar increase of cells in mitosis ( $p>0.05$, Fig. 2A and B). However, AMF was able to further increase the percentage of cells arrested in mitosis, which was in addition to PTX's effect in H-p53 $3^{\text {silenced }}$ cells $(3.4 \pm 0.7 \%$ in PTX vs. $7.6 \pm 0.9 \%$ in $\mathrm{P}+\mathrm{A}, \mathrm{p}<0.05)$, but AMF inhibited PTX-induced mitotic arrest in $\mathrm{H}$ cells (Fig. 2A). These results suggest that AMF enhances PTX-induced mitotic arrest in cancer cells with disrupted p53 expression.

The AMF modulation of PTX's effect on apoptosis is p53dependent. AMF enhancement of PTX-induced apoptosis was not observed in $\mathrm{H}$ cells (Fig. 3A), although PTX or AMF alone induced a significant increase of cells undergoing apoptosis when compared to the control $(4.6 \pm 0.3 \%$ in PTX or $2.9 \pm 0.4 \%$ in AMF vs. $1.7 \pm 0.2 \%$ in Ctrl, p<0.05, Fig. $3 \mathrm{~A}$ ). Again, knockdown of p53 in $\mathrm{H}$ cells restored the synergistic effect between these two drugs $(13.9 \pm 0.6 \%$ in P+A vs. $7.0 \pm 1.6 \%$ in PTX or $4.1 \pm 1.2 \%$ in AMF, Fig. $3 \mathrm{~B}$ ). Thus, $\mathrm{AMF}^{\prime}$ enhancement of PTX-induced apoptosis is also p53 statusdependent.

AMF and PTX modulation of cell cycle regulators, $p 21$ and Cdk1, is p53-dependent. AMF increased p21 protein levels in $\mathrm{H}$ cells but did not have a remarkable effect on p21 in CO and H-p53 $3^{\text {silenced }}$ cells (Fig. 4). This is consistent with the fact that p21 is a p53 target gene and regulation of p21 is p53dependent. This is also consistent with p21 as a negative
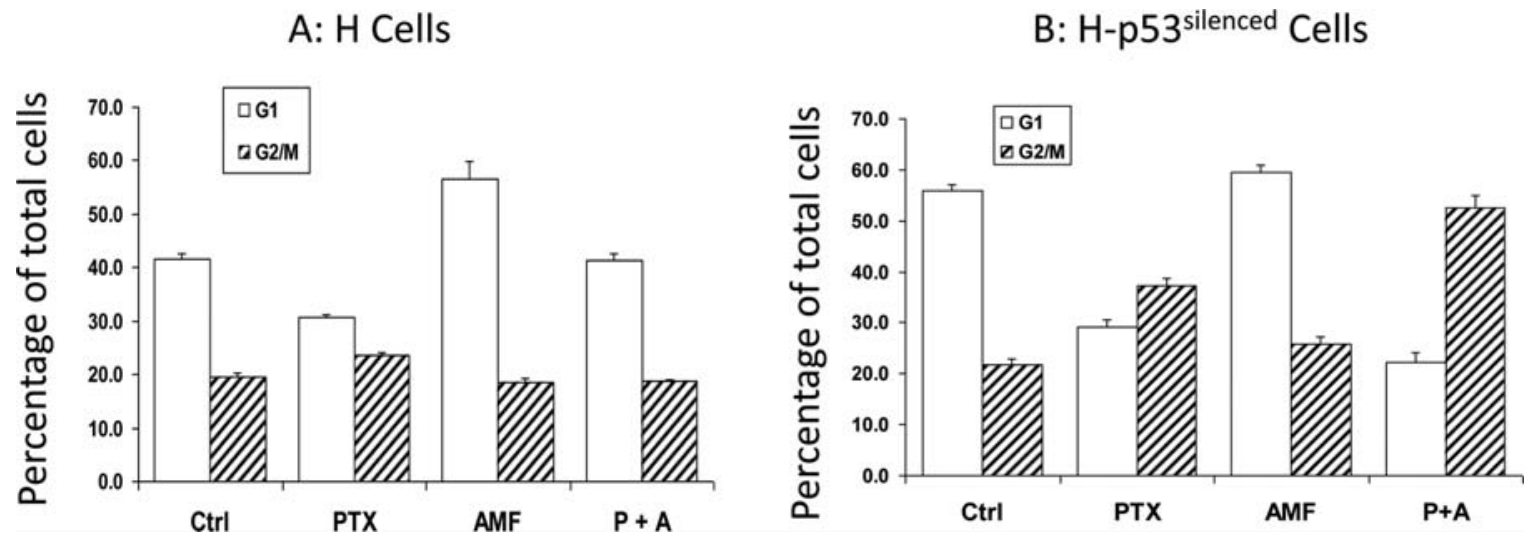

Figure 1. Paclitaxel and amifostine on the cell cycle. Cells were treated with vehicle (Ctrl) or $356 \mu \mathrm{M}$ amifostine (AMF) for $72 \mathrm{~h}$ or $14 \mathrm{nM}$ paclitaxel (PTX) for $24 \mathrm{~h}$, or both (P+A) $178 \mu \mathrm{M}$ amifostine for $72 \mathrm{~h}$ and $2 \mathrm{nM}$ paclitaxel for $24 \mathrm{~h}$ in cell culture dishes. Changes in the percentage of cells at the $\mathrm{G} 1$ or $\mathrm{G} 2 / \mathrm{M}$ phase were determined by a FacScan flow cytometry machine. (A) H cells. All comparisons were significantly different for the G1 phase except Ctrl vs. P+A $(\mathrm{p}<0.05)$. PTX is significantly different from Ctrl, AMF and $\mathrm{P}+\mathrm{A}$ in $\mathrm{G} 2 / \mathrm{M}$ phase $(\mathrm{p}<0.05)$. (B) H-p53 silenced cells. All comparisons were significantly different for $\mathrm{G} 1$ and $\mathrm{G} 2 / \mathrm{M}$ phase $(\mathrm{p}<0.05)$. 


\section{A: H Cells}

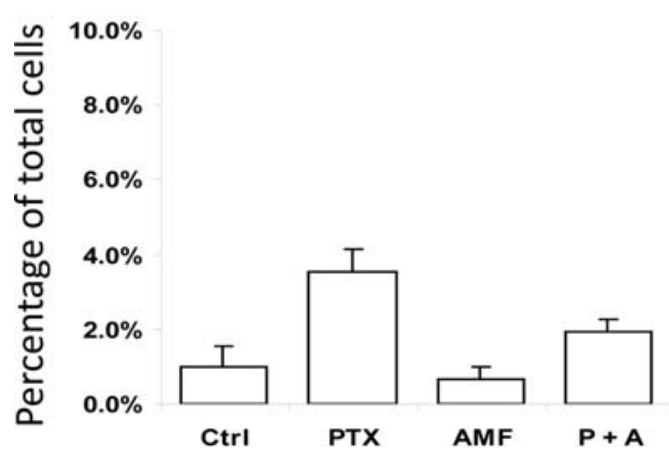

B: H-p53 $3^{\text {silenced }}$ Cells

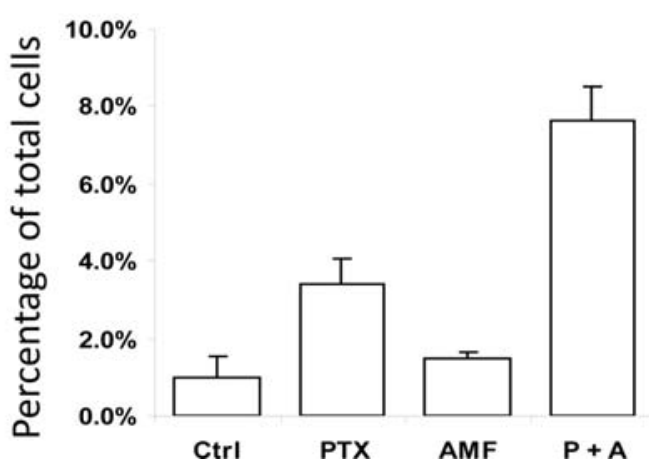

Figure 2. Paclitaxel and amifostine on cell mitosis. Cells were treated as at Fig. 1. Mitotic cells were identified by their characteristic chromatin morphology in the nuclei and counted under the microscope with a DAPI filter after stained with solution containing $3.7 \%$ formaldehyde, $0.6 \% \mathrm{NP} 40$, and $11 \mu \mathrm{g} / \mathrm{ml}$ Hoechst 33342. The ratio was obtained as a percentage of mitotic cells over total cell number in the same field. (A) H cells. The comparisons: PTX vs. all others and P+A vs. AMF were significantly different $(\mathrm{p}<0.05)$. (B) H-p53 silenced cells. All comparisons were significantly different $(\mathrm{p}<0.05)$ except Ctrl vs. AMF.

\section{A: H Cells}

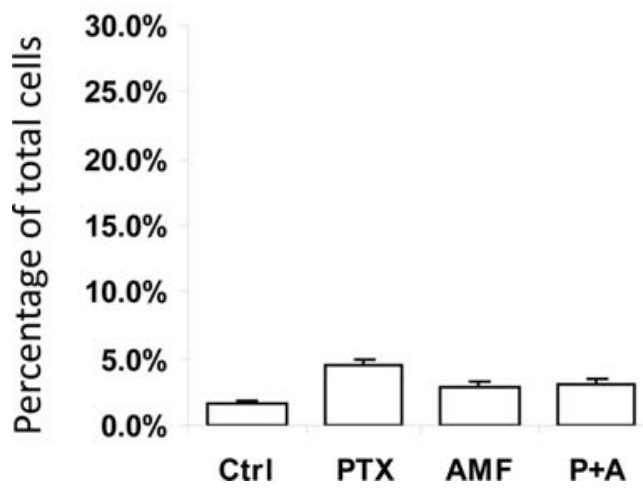

B: H-p53 $3^{\text {silenced }}$ Cells

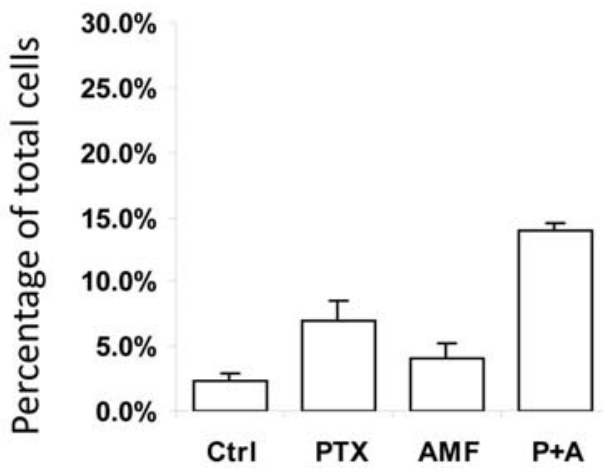

Figure 3. Paclitaxel and amifostine on cell apoptosis. Apoptotic cells were confirmed by their fragmented nuclear morphology and counted under the microscope with a DAPI filter. The ratio was obtained as a percentage of apoptotic cells over total cell number in the same field. (A) H cells. All comparisons were different except AMF vs. P+A (p<0.05). (B) H-p53 $3^{\text {silenced }}$ cells. All comparisons were different $(\mathrm{p}<0.05)$ except Ctrl vs. AMF and PTX vs. AMF. Means and SEs were calculated from at least three experiments, and analyzed by one-way ANOVA followed by Holm-Sidak method for pairwise comparison.

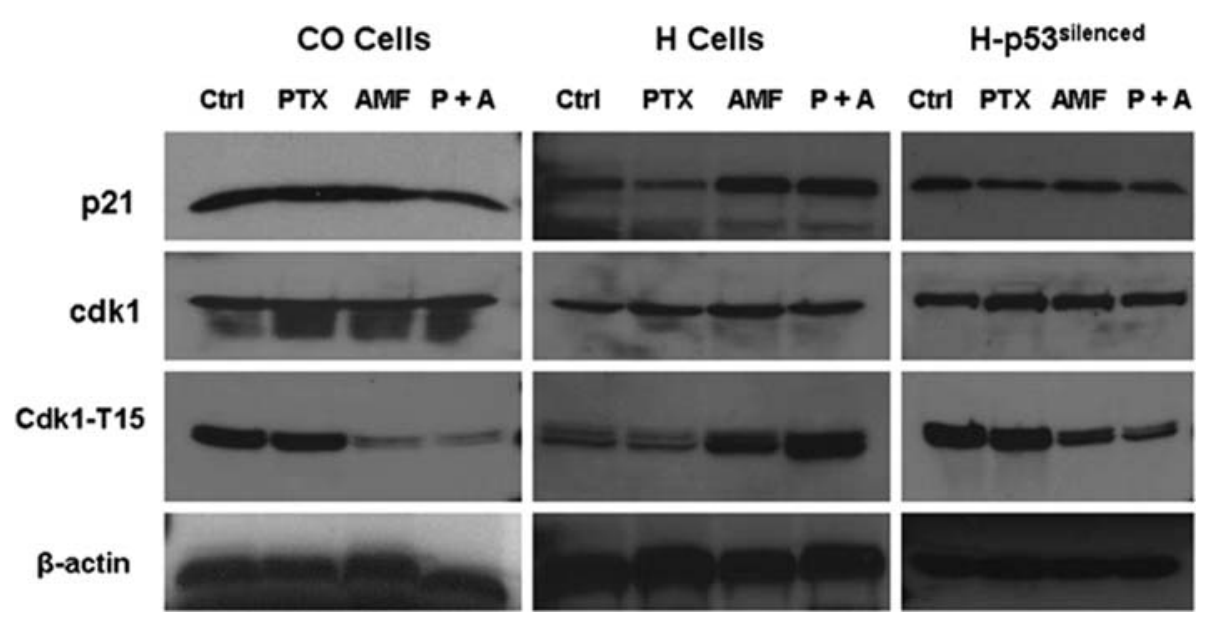

Figure 4. Paclitaxel and amifostine regulation of cell cycle proteins. Cells were treated with vehicle (Ctrl), amifostine (AMF, $356 \mu \mathrm{M}, 72 \mathrm{~h})$, paclitaxel (PTX, $14 \mathrm{nM}, 24 \mathrm{~h}$ ), or paclitaxel and amifostine (P, $2 \mathrm{nM}$ for $24 \mathrm{~h}$ and A, $178 \mu \mathrm{M}$ for $72 \mathrm{~h}$ ). Fifty micrograms of protein extract was loaded on SDS-PAGE gels and probed with primary antibodies against $\mathrm{p} 21$, total Cdk1, phosphorylated Cdk1 at Tyr15. B-actin was used as a loading control. 

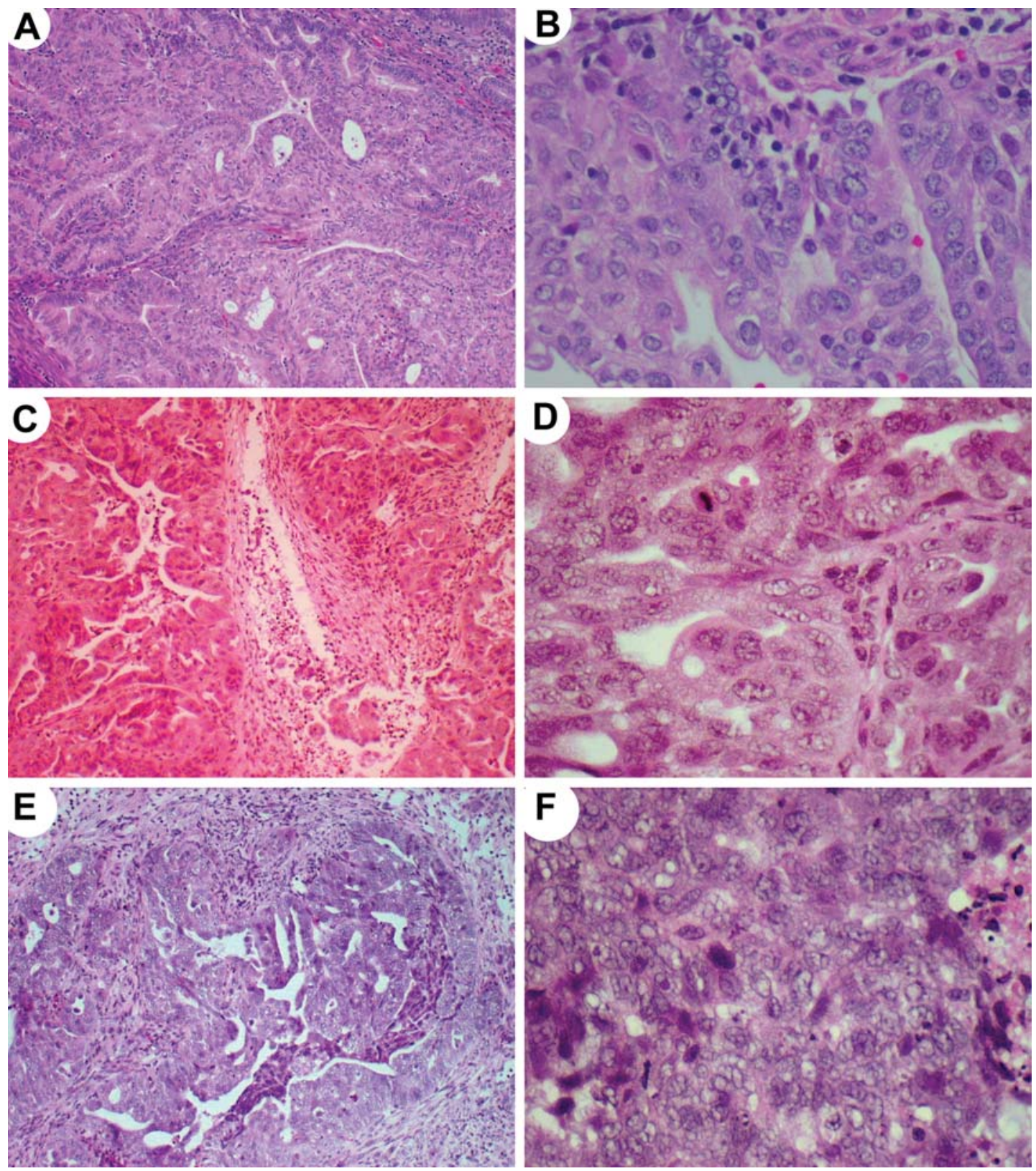

Figure 5. Histology of patient tumor and xenografts. Histology of the original patient tumor showing an endometrial carcinoma, endometrioid type, FIGO grade II (A and B) is compared with the histology of the patient tumor transplanted into athymic mice after one passage $(\mathrm{C}$ and $\mathrm{D})$ and five passages $(\mathrm{E}$ and $\mathrm{F})$. Hematoxylin and eosin stains, magnifications x100 (A, C and E) and x400 (B, D and F).

regulator of the G1-S transition since we observed a remarkable effect on G1 phase by AMF in H cells (Fig. 1B). Cdk1 is recognized as a conductor of the $\mathrm{M}$ phase (21). PTX and AMF did not change the total protein level of Cdk1 (Fig. 4), but AMF has been shown to differentially regulate the phosphorylation of Cdk1 at Tyr15 depending upon p53 status. AMF, either with or without PTX, increased the phosphorylation of Tyr15 in H cells (Fig. 4), indicating a reduced Cdk1 activity by AMF. When p53 expression was disrupted in $\mathrm{CO}$ and H-p53 $3^{\text {silenced }}$ cells, AMF induced a dephosphorylation of Tyr15 and consequently increased the kinase activity of Cdk1. This suggests that when p53 expression is disrupted, AMF enhances cancer cell sensitivity to PTX by facilitating cell entry into $M$ phase through activation of Cdk1. PTX alone did not have any effect on the phosphorylation status at Tyr15, suggesting that PTX regulation of Cdk1 activity, if any, involves mechanisms other than phosphorylation at Tyr15 in ECa cells.

Creation of xenografts from primary patient tumors in athymic mice. We succeeded in establishing xenografts in athymic mice using fresh tumors from endometrial cancer patients. Pathologic studies were conducted to compare tumor features among the original tumor and xenografts in mice (Fig. 5A-F). Tumor sections, stained with hematoxylin and eosin, show an endometrial carcinoma of the endometrioid type and an intermediate grade (FIGO grade II). The tumor was composed of glands and sheets of malignant cells and occasional papillary 
A

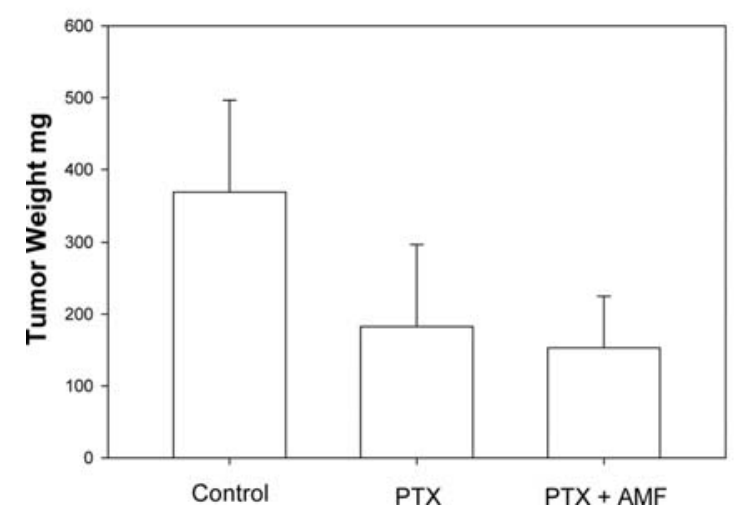

B

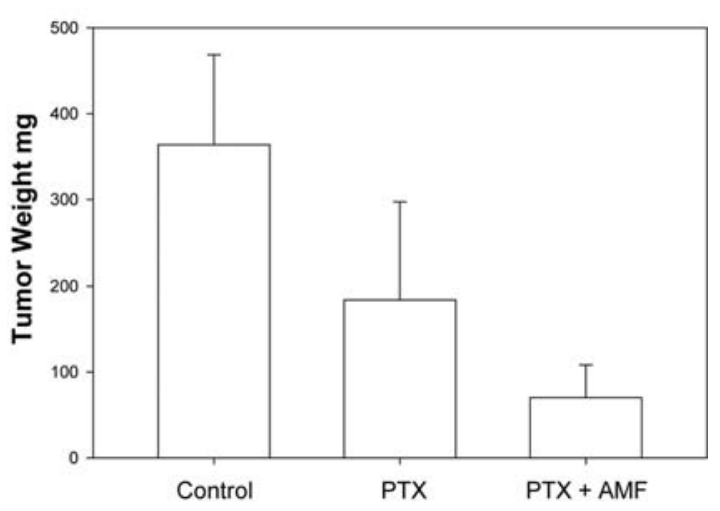

Figure 6. Paclitaxel and amifostine treatment of primary patient tumor xenografts. Creation of xenografts and treatment regimens were detailed in Materials and methods. Xenograft tumors isolated from the control (Control), treated with paclitaxel (PTX) and treated with both paclitaxel and amifostine (PTX+AMF) groups were weighted $(\mathrm{mg})$ and analyzed by one-way ANOVA followed by Holm-Sidak method for pairwise comparison. (A) Xenograft tumors created from a UEC patient tumors. PTX or PTX+AMF is significantly different from the Control ( $\left.{ }^{*} \mathrm{p}<0.05\right)$. (B) Xenograft tumors created from a UPSC patient tumor. All three groups are different from each other $(\mathrm{p}<0.05)$.

structures. Nucleoli were prominent. There was moderate nuclear pleomorphism and mitosis was rare. The histologic appearance of the original patient tumor was compared to the morphology of the tumor xenografts which had undergone up to 7 passages. These tumors resembled each other in terms of architectural pattern with abnormal glands, sheets and occasional papillary configurations as they would normally occur in intermediate grade endometrioid carcinomas. One difference between the original patient tumor and the xenografts was a higher number of mitotic figures found in the transplants. Tumor samples harvested from subcutaneous and intraperitoneal sites were also morphologically similar. These results indicate that the morphology of the original patient tumors was generally preserved in the xenografts.

TP53 status in patient tumors. After an initial successful xenograft growth in mice, two primary patient tumors were selected to further propagate into mice for the study of therapeutic effect of PTX and AMF. The first patient tumor was uterine endometrioid carcinoma (UEC) according to pathological diagnosis. DNA sequencing did not show any mutation in p53 coding region. The second tumor was uterine papillary serous carcinoma (UPSC) and sequencing showed a single substitution at the DNA binding domain at codon 209. The substitution of $\mathrm{T}$ for A resulted in creation of new stop codon of TGA from Arginine.

AMF did not enhance PTX's therapeutic effect on the UEC tumor which does not contain a mutation in the TP53 gene. The UEC tumor (first generation xenograft) was transplanted to athymic mice and the therapeutic effect of amifostine and paclitaxel on tumor growth was investigated (Fig. 6A). Amifostine was given at a dose of $120 \mathrm{mg} / \mathrm{kg}$ every Monday, Tuesday and Wednesday through intra-peritoneal injection, and paclitaxel was given at a dose of $12 \mathrm{mg} / \mathrm{kg}$ every Wednesday through tail vein injection. The control animals received only vehicle. The treatment was repeated every week for four weeks. The wet weights of subcutaneous tumors in paclitaxel treated animals $(n=12)$ were significantly less than in animals receiving vehicle $(182.7 \mathrm{mg} \pm 114.1, \mathrm{n}=12 \mathrm{vs}$. $368.6 \mathrm{mg} \pm 128, \mathrm{n}=11, \mathrm{p}<0.05)$. Pretreatment of amifostine followed by paclitaxel, however, did not change tumor weight (152.9 mg $\pm 71.8, \mathrm{n}=12)$ significantly when compared to paclitaxel alone $(\mathrm{p}=0.50)$ although the tumor sizes with PTX and AMF are significantly smaller than the control $(\mathrm{p}>0.05)$. This study suggests that AMF did not provide any additional benefit in tumors treated with PTX.

AMF significantly enhanced PTX's therapeutic effect on UPSC with a p53 mutation. We also investigated the effect of amifostine on paclitaxel's control of tumor growth in the second tumor, a UPSC with a p53 point mutation. Paclitaxel and amifostine treatment was administered as described above. The wet weights of subcutaneous tumors in paclitaxel treated animals were significantly less than in animals receiving vehicle $(183.9 \pm 113.4 \mathrm{mg}, \mathrm{n}=13$, vs. $363.8 \pm 104.7 \mathrm{mg}, \mathrm{n}=12$, $\mathrm{p}<0.05$, Fig. 6B). Addition of amifostine to paclitaxel treatment $(n=13)$ further reduced tumor weight to $70.0 \pm 38.3 \mathrm{mg}$, which is significantly less than paclitaxel treatment alone $(\mathrm{p}<0.05$, Fig. 6B). This study suggests that, as in cells grown in vitro, amifostine enhances the anti-cancer effect of paclitaxel in xenografted primary UPSC with a mutation in the TP53 gene.

\section{Discussion}

We have previously reported that AMF not only can enhance PTX's direct effect on tumor growth in athymic mice but also can prolong animal survival (12). Additionally, AMF can protect mouse red blood cells and white blood cells from side effects of PTX (12). However, these studies were conducted with an endometrial cancer cell line, Hec50co cells, which contain a TP53 null mutation (15). Additionally, previous in vivo studies were conducted in xenografts created from Hec50co cells. While these studies did suggest that AMF enhances paclitaxel's therapeutic effect in cancer cells, it was not clear if their synergistic effect was dependent upon the cell line we used or related to the subtype of endometrial cancer 
and TP53 status. In order to investigate the underlying mechanism for the synergy between AMF and PTX, we created a p53 protein knockdown cell from Ishikawa $\mathrm{H}$ cell, which represents type I endometrial cancer and is believed to have a functional p53 protein $(14,15)$.

Indeed, synergy between AMF and PTX was observed after p53 knockdown in Ishikawa $\mathrm{H}$ cells. The synergy was shown in the following ways: i) the regulation of cell cycle, ii) induction of apoptosis, and ii) the reduction in the $\mathrm{IC}_{50}$ value for PTX in p53 $3^{\text {silenced }}$ cells (Table I). The knockdown of p53 in Ishikawa $\mathrm{H}$ cells changed the cell phenotype to more resemble another cell line with endogenous mutant and nonfunction p53, Hec50co cells. Thus, it is clear that p53 status controls drug sensitivity and predicts for the ability of AMF to synergize with PTX as shown in Fig. 1.

In parental Ishikawa $\mathrm{H}$ cells, AMF up-regulated p21 and increased Cdk1 phosphorylation at tyrosine 15 , which resulted in $\mathrm{G} 1$ phase arrest and reduced entry into G2-M phases (Fig. 1A). By contrast, when $\mathrm{p} 53$ was knocked down in $\mathrm{H}$ cells, The AMF effect on G1 phase was modest albeit significant (Fig. 1B), whereas it resulted in a significant induction of the percentage of cells in $\mathrm{G} 2-\mathrm{M}$ phase $(25.9$ vs. $21.8 \%$, $\mathrm{p}<0.05)$. The AMF effect on G2-M phase was much more remarkable in cells receiving both AMF and PTX, and significantly higher than in cells treated with PTX alone (Fig. 1B). Cdk1 was shown to be dephosphorylated, similar to that observed in Hec50co cells (Fig. 4).

Thus, it seems that there are two pathways that mediate AMF effects. G1 phase arrest by AMF is likely to be mediated through p21 upregulation in a p53-dependent manner (Fig. 4). In cells with dysfunctional p53, an alternative AMF pathway appears to be mediated through activation of Cdk1 kinase, resulting in increased entry into G2-M phase. PTX binds to the B-tubulin subunit and promotes tubulin polymerization, which stabilizes the microtubules. This interferes with the normal function of the mitotic spindle during cell mitosis, resulting in mitotic arrest at the metaphase/anaphase junction, which triggers the mitotic spindle checkpoint and consequently apoptosis (22). Increased entry into G2-M phase induced by AMF makes cancer cells more susceptible to PTX action, thus enhancing PTX's therapeutic effects. Such dual regulation of cell cycle by AMF can be explored in some subtypes of endometrial cancer. These findings are particularly relevant for the subtype of endometrial cancer, UPSC, with high prevalence of p53 mutations $(10,11)$. Our previous report showed that a combinatorial regimen with PTX and AMF is effective in vivo using a mouse xenograft created by transplantation of Hec50co cells, a verified model for UPSC $(15,16)$. We have shown here that this regimen is equally effective in patient tumors harboring a dysfunctional p53 mutation.

While cancer cell lines readily generate xenografts, they may carry excess genetic alterations not normally present in patient tumors. We created primary patient tumor xenografts in order to preserve the original morphologic and genetic features. We have shown that patient tumors generally preserve their morphology and tumor differentiation after several passages.

We selected two patient tumors, one with and one without a dysfunctional p53 mutation. The mutation occurred at codon 209 , with a substitution of $\mathrm{T}$ for $\mathrm{A}$. This resulted in a premature stop codon. While the growth of these two tumors and their response to PTX alone is not significantly different, their response to AMF and PTX was significantly different (Fig. 6). AMF did not show any additive effect when used with PTX in the tumor without a p53 mutation, but in the tumor with the dysfunctional p53 mutation, AMF significantly enhanced the PTX anticancer effect.

Our study proposes a novel therapeutic strategy in pooroutcome endometrial cancer, using amifostine to sensitize tumors with TP53 mutations to paclitaxel. This strategy has now been validated in primary patient tumor xenografts herein and strongly supports a future clinical trial in endometrial tumors with dysfunctional p53.

\section{Acknowledgements}

We thank Ms. Anna Holmes and Mr. Tan Ngyen for their contributions in performing experiments essential for this report. This study was supported in part by American Cancer Society Research Scholar Grant RSG-06-105-01CCE (DD).

\section{References}

1. American Cancer Society: Cancer Facts and Figures. American Cancer Society, 2009.

2. Rose PG: Endometrial carcinoma. N Engl J Med 335: 640-649, 1996.

3. DiSaia PJ and Creasman WT: Adenocarcinoma of the Uterus. In Clinical Gynecologic Oncology. DiSaia PJ and Creasan WT (eds). Mosby, Inc., pp137-172, 2002.

4. Sherman ME: Theories of endometrial carcinogenesis: a multidisciplinary approach. Mod Pathol 13: 295-308, 2000.

5. Rosenberg P, Risberg B, Askmalm L and Simonsen E: The prognosis in early endometrial carcinoma. The importance of uterine papillary serous carcinoma (UPSC), age, FIGO grade and nuclear grade. Acta Obstet Gynecol Scand 68: 157-163, 1989.

6. Rosenberg P, Blom R, Hogberg T and Simonsen E: Death rate and recurrence pattern among 841 clinical stage I endometrial cancer patients with special reference to uterine papillary serous carcinoma. Gynecol Oncol 51: 311-315, 1993.

7. Hendrickson M, Ross J, Eifel P, Martinez A and Kempson R: Uterine papillary serous carcinoma: a highly malignant form of endometrial adenocarcinoma. Am J Surg Pathol 6: 93-108, 1982.

8. Chambers JT, Merino M, Kohorn EI, Peschel RE and Schwartz PE: Uterine papillary serous carcinoma. Obstet Gynecol 69: 109-113, 1987.

9. Berchuck A and Boyd J: Molecular basis of endometrial cancer. Cancer 76: 2034-2040, 1995.

10. Tashiro H, Isacson C, Levine R, Kurman RJ, Cho KR and Hedrick L: p53 gene mutations are common in uterine serous carcinoma and occur early in their pathogenesis. Am J Pathol 150: 177-185, 1997.

11. Lax SF, Kendall B, Tashiro H, Slebos RJ and Hedrick L: The frequency of p53, K-ras mutations, and microsatellite instability differs in uterine endometrioid and serous carcinoma: evidence of distinct molecular genetic pathways. Cancer 88: 814-824, 2000.

12. Dai D, Holmes AM, Nguyen T, Davies S, Theele DP, Verschraegen $\mathrm{C}$ and Leslie KK: A potential synergistic anticancer effect of paclitaxel and amifostine in endometrial cancer. Cancer Res 65: 9517-9524, 2005.

13. Kumar NS, Richer J, Owen G, Litman E, Horwitz KB and Leslie KK: Selective down-regulation of progesterone receptor isoform B in poorly differentiated human endometrial cancer cells: implications for unopposed estrogen action. Cancer Res 58: 1860-1865, 1998 .

14. Albitar L, Carter MB, Davies S and Leslie KK: Consequences of the loss of p53, RB1, and PTEN: relationship to gefitinib resistance in endometrial cancer. Gynecol Oncol 106: 94-104, 2007. 
15. Albitar L, Pickett G, Morgan M, Davies S and Leslie KK: Models representing type I and type II human endometrial cancers: Ishikawa $\mathrm{H}$ and Hec50co cells. Gynecol Oncol 106 $52-64,2007$.

16. Dai D, Albitar L, Nguyen T, Laidler LL, Singh M and Leslie KK A therapeutic model for advanced endometrial cancer: Systemic progestin in combination with local adenoviral-mediated progesterone receptor expression. Mol Cancer Ther 4: 169-175, 2005.

17. Meng X, Lu H and Shen Z: BCCIP functions through p53 to regulate the expression of p21Waf1/Cip1. Cell Cycle 3: 1457-1462, 2004.
18. Meng X, Yuan Y, Maestas A and Shen Z: Recovery from DNA damage-induced $\mathrm{G} 2$ arrest requires actin-binding protein filamin-A/actin-binding protein 280. J Biol Chem 279: 6098-6105, 2004.

19. Nishida M: The Ishikawa cells from birth to the present. Hum Cell 15: 104-117, 2002.

20. Jordan MA and Wilson L: Microtubules as a target for anticancer drugs. Nat Rev Cancer 4: 253-265, 2004.

21. Nigg EA: Mitotic kinases as regulators of cell division and its checkpoints. Nat Rev Mol Cell Biol 2: 21-32, 2001.

22. Bhalla KN: Microtubule-targeted anticancer agents and apoptosis. Oncogene 22: 9075-9086, 2003. 